P03

A CURIOUS CASE OF AUTOIMMUNE HEPATITIS AND ACQUIRED PARTIAL LIPODYSTROPHY

${ }^{1}$ Christopher Bakewell, ${ }^{1}$ Astor Rodrigues, ${ }^{2}$ Rachel Williams, ${ }^{3}$ Girish Gupte, ${ }^{1}$ Kavinda Dayasiri, ${ }^{1}$ Geetha Anand. 'Oxford University Hospitals NHS Foundation Trust; ${ }^{2}$ Cambridge University Hospitals NHS Foundation Trust; ${ }^{3}$ Birmingham Women's and Children's Hospital NHS Foundation Trust

10.1136/flgastro-2021-bspghan. 14

Background The lipodystrophies are an extremely rare group of metabolic conditions. In acquired partial lipodystrophy (APL), the predominant clinical feature is a progressive, symmetrical reduction of adipose tissue which typically begins during the pre-adolescent period. While the pathogenesis of APL is likely to be complex and heterogeneous, a subset of these patients are reported to develop associated autoimmune conditions, including hepatitis. This has led some to implicate an underlying autoimmune process.

Method We report an ultra-rare case of a 17-month-old boy who presented to hospital with a Henoch-Schönlein Purpuralike illness and subsequently developed anti-LKM-1 positive autoimmune hepatitis (AIH) as well as clinical features of Acquired Partial Lipodystrophy.

Results In this child, the features of lipodystrophy occurred at a very early age and in association with a relatively mild hepatitis. We document his challenging path from first presentation, through to the diagnosis of $\mathrm{AIH}$ and APL and discuss his ongoing management. Despite an encouraging reduction in liver transaminases following dual immunosuppression, no improvement was observed in his adipose tissue distribution.

Conclusions Cases of autoimmune hepatitis occurring in association with acquired partial lipodystrophy are extremely rare. The primary aim of this report is therefore to familiarise the PGHAN community with this uncommon association. While much is still unknown about the link between these conditions, we use this case to discuss the current evidence for their shared pathogenesis.

\section{P04 A DIAGNOSTIC DILEMMA: CASE REPORT OF A YOUNG BOY WITH ABDOMINAL TUBERCULOSIS WHO WAS INITIALLY THOUGHT TO HAVE CROHN'S DISEASE}

Alice Findlay, Natasha Burgess, Marco Gasparetto, Ahmed Kadir, Sandhia Naik, Nick Croft, Protima Deb. The Royal London Hospital

\subsection{6/flgastro-2021-bspghan. 15}

Introduction Tuberculosis (TB) like Crohn's disease can affect any part of the gastro-intestinal (GI) tract including anus, peritoneum and hepato-biliary system. The clinical manifestations of abdominal tuberculosis are non-specific and can mimic various GI disorders especially Crohn's disease which can cause delay in diagnosis and management.

History and Presentation A 14-year-old boy was diagnosed with small bowel ileal Crohn's disease in 2016 based on clinical symptoms of abdominal pain and weight loss, biochemical features of a raised ESR but normal CRP at presentation and a distorted ileocaecal valve (ICV) with inflammatory changes seen both macroscopically and microscopically at colonoscopy with radiological confirmation of short segment ileal disease on MRI. He was treated with exclusive enteral nutrition for induction of remission, however his ESR remained elevated and he required escalation to Azathioprine within 3 months of diagnosis for continued symptoms of abdominal pain and ongoing weight loss. His clinical course over the next 2 years remained unchanged with a persistently raised ESR and continued disease around ICV and distal ileum in spite of immunomodulator therapy.

Treatment and Investigation Prior to commencing biologic treatment for active Crohn's disease, he had an Elispot and was found to be positive. This was felt to be consistent with latent $\mathrm{TB}$ infection for which he had 3 months of chemoprophylaxis with Rifampicin and Pyridoxine. Following this, his symptoms of abdominal pain resolved, and he gained $5 \mathrm{~kg}$ for the first time since his diagnosis of CD. Moreover, his ESR completely normalised. His repeat mri showed a significant improvement of the inflammation in the ileum as well as around the ICV. This was also confirmed with repeat colonoscopy which was markedly improved from previously although still had abnormal distortion of the ICV. His clinical response to the TB treatment and radiological and endoscopic improvement following the $\mathrm{TB}$ chemoprophylaxis led to the suspicion of intestinal TB as the correct diagnosis.

Clinical Background and Progress

He was born in the UK. He had a BCG scar. His grandmother was diagnosed with TB in India in 2010. She had visited the UK prior to the diagnosis and stayed with the family for 6 months. She was unwell with cough and weight loss at that time. Both his mother and father had been exposed to her also and his father was also receiving treatment for latent TB now. Based on the history of TB exposure and the clinical, biochemical, endoscopic and radiological improvement following latent $\mathrm{TB}$ treatment, he went on to complete a full 6-month course with 4 drug initiation for abdominal TB.

Summary and Conclusion Abdominal tuberculosis should be considered as a differential diagnosis in patients with Crohn's disease. Careful evaluation of clinical, biochemical, radiological and histological findings can aid in distinguishing between the two conditions, leading to early diagnosis and management.

\section{P05 A SERVICE EVALUATION OF A DIETETIC-LED CLINIC FOR THE MANAGEMENT OF PAEDIATRIC PATIENTS WITH COELIAC DISEASE}

Isobel Connolly. CHI Ireland at Crumlin

10.1136/flgastro-2021-bspghan.16

Introduction The management of Coeliac disease (CD) is through a gluten free diet (GFD). The current BSPGHAN guidelines advise that annual follow-up with both a paediatric dietitian and paediatric gastroenterologist is necessary to ensure continued adherence to a GFD.

However there are many NHS trusts where paediatric patients with $\mathrm{CD}$ are only reviewed in a dietetic-led clinic (DLC) annually.

Aims and Objectives To evaluate a DLC for the management of paediatric patients with $\mathrm{CD}$ two years after its establishment. In the current service patients are reviewed on an annual basis by the dietitians after an initial post-diagnosis appointment with a paediatric gastroenterologist and if identified as requiring a medical review through the 'red flag' criterion they will be referred back to the paediatric 
gastroenterologist. The objectives of this service evaluation were to assess satisfaction rates with the DLC and establish GFD compliance rates of patients attending the DLC.

Subjects and Methods Patients who had attended the DLC in the previous year were invited to partake in the service evaluation. The method was two online questionnaires. The satisfaction questionnaire had previously been utilised in the same centre to assess satisfaction with the service prior to the change to a DLC service. The dietary compliance questionnaire had previously been utilised in another paediatric study.

Results The patient population response rate was $40 \%$ $(n=28) .61 \%$ of respondents were 'extremely likely' to recommend the DLC to friends and family if similar care was needed. $61 \%$ of respondents were 'fully compliant', 32\% of respondents were 'compliant with errors' and 7\% of respondents were 'non-compliant' with a GFD. 79\% were 'fully compliant' in the age-group 3-11 years compared to $43 \%$ in the age-group 12-18 years. Chi-square analysis showed this difference was approaching statistical significance $(p=0.053)$. The responses to the question 'My child eats food labelled 'May contain traces of gluten or wheat'" stratified by the two agegroups showed that $86 \%$ of respondents in the age-group 311 years responded 'no' whereas 50\% of the respondents in the age-group 12-18 years responded 'no' to this question. Chi-square analysis showed that this difference reached statistical significance $(p=0.043)$.

Summary and Conclusion A comparison between the satisfaction survey results performed prior to the service change shows that there is an increased proportion of respondents from the DLC service who were 'extremely likely' to 'recommend the service to a friend or family member' (61\%) compared to the previous service (38\%). During the two year period since the DLC service has been in place, two patients from 70 patients who are reviewed in the DLC required referral to the gastroenterologist. Significant financial savings are associated with a DLC compared with the previous service. The compliance rates of the overall population group and the lower compliance rates in the adolescent sub-group found are in accordance with the available literature. The questionnaire was able to identify some areas where adolescents were falling down in their compliance to a GFD and this informed the development of an online education resource.

\section{P06 A SINGLE CENTRE DESCRIPTION OF IBD PATIENTS WITH NEGATIVE FAECAL CALPROTECTIN AT DIAGNOSIS}

Harween Dogra, Vinod Kolimarala, Bukunola Kukoyi, Babu Vadamalayan. King's College Hospital

\subsection{6/flgastro-2021-bspghan.17}

Introduction In the past 10 years faecal calprotectin has been increasingly used in Paediatrics, as in adults, to screen for inflammatory bowel disease (IBD). Faecal calprotectin is a calcium and zinc binding protein expressed by neutrophils and can be detected in stool when there is infiltration of the mucosa with neutrophils, as in IBD. Discussion around diagnostic values in Paediatrics have shown young children can have falsely elevated faecal calprotectin and studies have suggested values over 200 , or some over 800 , as a positive indication to investigate further for IBD. However, there are limited descriptions of IBD patients with negative faecal calprotectin and how this may influence investigation and management.
Methods We looked at retrospective data 20011-2020 of our IBD case load to identify how many had a negative/low faecal calprotectin (low FC) at diagnosis, this limit was set at $<80$, which is the current value for our hospital laboratory. Positive/raised faecal calprotectin (high FC) was any value $>80$. Our local database was used to identify IBD patients and the hospital electronic patient records system was searched for faecal calprotectin values at time of diagnosis (pre-treatment).

Results A total of 198 patients were diagnosed with IBD in the time frame investigated. $17(9 \%)$ patients had a negative faecal calprotectin at diagnosis, $118(60 \%)$ had a positive value, and 63 (32\%) had no documented value (figure 1). The difference in FC values was significantly different between the low FC and high FC group, $\mathrm{p}<0.0001$ (Mann-Whitney test, figure 3$)$. The median age at diagnosis was 14 yrs (2-16 yo) in the low FC group, and 12 yrs (1-17 yo) in the high FC group (figure 2). The distribution of IBD diagnosis in the

Total IBD patients 198 I

Low FC 17 High FC 118 Not documented 63

Abstract P06 Figure 1 Total of 198 IBD patient reviewed, 63 with no documented FC (faecal calprotectin), 17 with low/normal FC and 118 with high/raised FC

\begin{tabular}{|l|l|l|}
\hline & Low FC & High FC \\
\hline Age, median (range) & $14(2-16)$ & $12(1-17)$ \\
\hline Crohn's Disease & 5 & 61 \\
\hline Ulcerative colitis & 6 & 43 \\
\hline IBD-U & 6 & 14 \\
\hline
\end{tabular}

Abstract P06 Figure 2 Median age of patients with low/normal FC (faecal calprotectin) is 14yo, 5 patients with Crohn's disease, 6 patients with Ulcerative colitis, 6 patients with IBD-U. Median age of patients with high/raised FC is 12yo, 61 patients with Crohn's disease, 43 patients with Ulcerative colitis, 14 patients with IBD-U

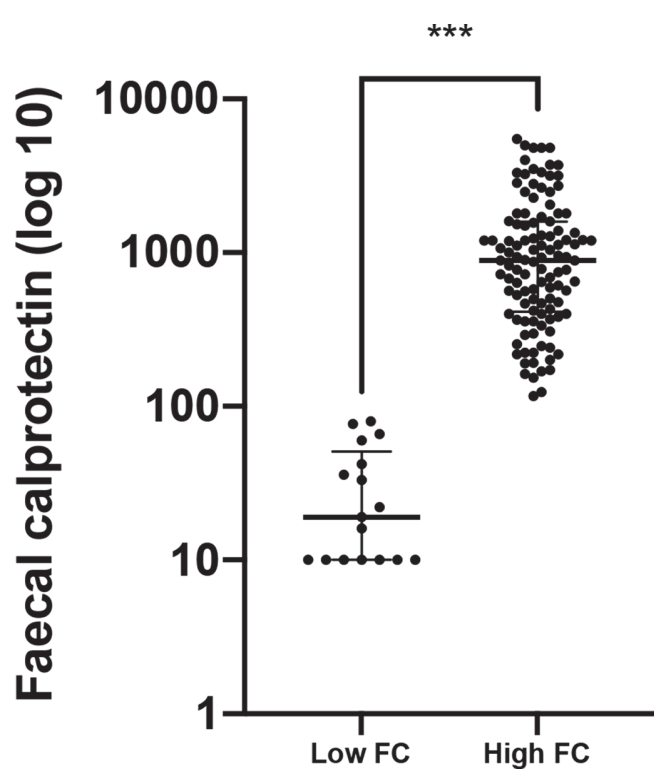

Abstract P06 Figure 3 There was a significant difference in FC (Faecal calprotectin) values between the normal/low FC and raised/high FC groups, ${ }^{* * *} p<0.0001$ (Mann-Whitney test) 